\title{
Medial temporal lobe damage impairs representation of simple stimuli
}

\section{David E. Warren ${ }^{1 *}$, Melissa C. Duff ${ }^{2}$, Daniel Tranel ${ }^{2}$ and Neal J. Cohen ${ }^{1}$}

1 Beckman Institute, University of Illinois at Urbana-Champaign, Urbana, IL, USA

2 Department of Neurology, University of lowa, lowa City, IA, USA

Edited by:

Russell A. Poldrack, University of

California, USA

Reviewed by:

Morgan Barense, University of Toronto,

Canada

lan G. Dobbins, Duke University, USA

*Correspondence:

David E. Warren, Department of

Neurology, University of lowa, 200

Hawkins Drive, 2100 RCP, lowa City,

lowa 52240, USA.

e-mail: davideugenewarren@gmail.com
Medial temporal lobe (MTL) damage in humans is typically thought to produce a circumscribed impairment in the acquisition of new enduring memories, but recent reports have documented deficits even in short-term maintenance. We examined possible maintenance deficits in a population of MTL amnesics, with the goal of characterizing their impairments as either representational drift or outright loss of representation over time. Patients and healthy comparisons performed a visual search task in which the similarity of various lures to a target was varied parametrically. Stimuli were simple shapes varying along one of several visual dimensions. The task was performed in two conditions, one presenting a sample target simultaneously with the search array and the other imposing a delay between sample and array. Eye-movement data collected during search revealed that the duration of fixations to items varied with lure-target similarity for all participants, i.e., fixations were longer for items more similar to the target. In the simultaneous condition, patients and comparisons exhibited an equivalent effect of similarity on fixation durations. However, imposing a delay modulated the effect differently for the two groups: in comparisons, fixation duration to similar items was exaggerated; in patients, the original effect was diminished. These findings indicate that MTL lesions subtly impair short-term maintenance of even simple stimuli, with performance reflecting not the complete loss of the maintained representation but rather a degradation or progressive drift of the representation over time.

Keywords: memory, STM, WM, MTL, hippocampus, delay, decay, amnesia

\section{INTRODUCTION}

Medial temporal lobe (MTL) lesions cause profound impairments in the ability to combine arbitrarily related information in an enduring fashion (Scoville and Milner, 1957; Cohen and Squire, 1980; Cohen and Eichenbaum, 1993; Eichenbaum and Cohen, 2001), but are typically thought to leave other cognitive and perceptual processes intact. This consensus view has been challenged recently by demonstrations of impaired maintenance of stimuli by MTLlesion patients over very short delays (e.g., Ryan and Cohen, 2004a; Hannula et al., 2006, 2007). In most reports the stimuli that present unusual difficulty are complex (e.g., Buffalo et al., 1998; Nichols et al., 2006; Hartley et al., 2007), but even some relatively simple items including colors (Olson et al., 2006a) and shapes (Sidman et al., 1968) have exposed impairment. These findings collectively indicate that MTL structures contribute to representations useful not only for subsequent memory performance but also for online processing. However, for the most part these reports did not attempt to characterize the representations still available, or the nature of the representational impairment, when the MTL had been damaged. Our investigation was implemented with the goal of providing further insight into the quality of representations that MTL-lesion patients can maintain across a delay.

Early scientific writing about MTL-lesion patients tended to support the idea that they had an intact capacity for the brief maintenance of information sufficient to support normal online processing, e.g., "to a casual observer this man seems like a relatively normal individual, since his understanding and reasoning are undiminished" (Scoville and Milner, 1957). The gross nature of the deficit in enduring memory was an obvious and crucial target for research, and exploration of that impairment proved immensely valuable to the field. Meanwhile, even sophisticated investigations of the remaining short-term memory capacity of patients like H.M. generally concluded that MTL lesions did not impair maintenance of simple stimuli, and many subsequent reports continued to support that conclusion. Published work from this era and afterward demonstrated that MTL lesions did not interfere with normal maintenance of consonant trigrams, sets of one- and three-digit numbers (Sidman et al., 1968), pure tones (Wickelgren, 1968), numbers used in digit-span tasks, judgments of the number of dots in a display, single relative spatial locations, angles between line segments, mirror-reversed arrays of squares (Cave and Squire, 1992), and multiple spatial locations (Warrington and Baddeley, 1974) among many others. Results of this nature indicated that maintenance of many kinds of stimuli is unimpeded by MTL damage.

However, counter-examples are available from that early phase of neuropsychological investigation (e.g., Prisko, 1963; Sidman et al., 1968). One underappreciated study from that time, by Sidman et al. (1968), employed simple shapes and provided a forced-choice test format whose lures were drawn from a continuum of items similar to the maintained target. On each trial, the participant was presented with a sample ellipse of a given eccentricity, followed by an imposed delay that varied by condition, and then a search 
array consisting of eight ellipses of varying eccentricity. Each search array included a target item which matched the eccentricity of the sample along with seven lures. Findings showed that when the sample ellipse and search array were presented simultaneously, H.M. selected the target item almost exclusively, but as the length of the delay increased so did the likelihood of selection of a lure rather than of the target.

Two distinct predictions could be made a priori about the distribution of H.M.'s responses in these delayed-match trials, with very different implications. If his errors arose from representations occasionally being lost during the delay, slipping from consciousness altogether, then endorsement of lures would be distributed randomly on those trials. If, however, errors arose from a maintenance process that was defective but did not suffer all-or-none loss, then a degraded representation might persist and lures similar to the sample would be selected disproportionately. This second pattern of results was observed for H.M., which differed substantially from the pattern exhibited by comparison participants (consisting of two children, more on this below). Looking at performance for the different delay conditions, H.M.'s responses drifted further and further from the original sample whereas those of comparison participants did not show any sign of representational change with increased delay.

In the last decade, findings of deficient maintenance have been more widely reported. Faces (Nichols et al., 2006; Olson et al., 2006a), other complex objects (Holdstock et al., 2000; Lee et al., 2005; Barense et al., 2007), spatial arrays (Olson et al., 2006b), scenes (Ryan et al., 2000; Ryan and Cohen, 2004a,b; Lee et al., 2005; Hannula et al., 2006), topographical maps (Hartley et al., 2007), and arbitrary pairings (Hannula et al., 2007) have all revealed impaired maintenance in patients with MTL damage. However, while the implications of these reports for maintenance are clear, none employ stimuli or methods that permit inferences to be drawn about the quality of any residual representation in incorrect trials and hence about the nature of the impairment.

Notably, most studies showing deficits in short-term maintenance employed stimuli substantially more complex than those employed by Sidman et al. (1968). This difference in materials may be important in reconciling the early indications of unimpaired maintenance with more recent reports of deficits. Even when MTL is damaged, simple stimuli may be represented and maintained sufficiently well to permit superficially normal performance if lures and targets are not similar to one another. By contrast, complex stimuli may not afford representations or maintenance that would allow normal performance under any circumstances. Concisely, test sensitivity may be the issue. By this hypothesis, simple items would appear to be maintained normally in all but the most sensitive tests, while complex stimuli would reveal performance deficits even in tests with low sensitivity, but in both cases the maintained representation would be deficient.

Our current work was intended to explore the nature and quality of briefly maintained representations of simple stimuli and the consequences of MTL damage for those representations. We asked MTL-lesion patients and healthy comparison participants (henceforth, "patients" and "comparisons") to perform a visual search task in two conditions: simultaneously, in which the sample item and search array were displayed at the same time; and delayed, wherein the sample was presented briefly and then removed for several seconds before the search array was presented. In all cases, the search array contained items comprising a continuum along a visual dimension, e.g., orientation, with a target item being an exact match to the sample item and a set of lures differing in systematic steps from the sample item. Six different visual dimensions were tested in this fashion. Behavioral responses were collected during the search task, and we also recorded eye movement data during each session. We anticipated that eye movements and fixations might vary with lure-target similarity, and that such variation could be used as an index of the quality of the maintained representations. Additionally, because multiple items could be fixated during each test trial, eye movements could provide a rich and efficient source of information. Although eye movements are a covert expression of prior experience, we did not anticipate that they would reflect implicit memory; rather, we sought to add a highly sensitive dependent measure to our task.

Specific predictions can be made about the variation by condition of any measure that indexes lure-sample similarity and thereby informs us about the maintained representations. If MTL damage produces deficits in the maintenance even of simple stimuli, MTL-lesion patients and comparisons should perform similarly in the simultaneous condition, and then differences should emerge in the delay condition. Failures of maintenance could potentially involve complete loss of stimulus representations, in which case measures sensitive to lure-sample similarity would reflect essentially random sampling of the search array; or failures might be the result of degradation or drift of a representation over time, in which case the same measures would instead reflect an attenuated relation between lure and sample similarity. Notably, random sampling of the search array would not necessarily imply complete loss of a representation, but would indicate a rapid degradation of the representation and corresponding lack of influence on an extremely sensitive and efficient dependent measure.

\section{MATERIALS AND METHODS PARTICIPANTS}

Eight neurological patients (three female) and eight matched comparison participants were included in the current study (see Table 1). The patients had varying etiologies each resulting in damage to MTL structures and dense amnesia: five were amnesic secondary to anoxic episodes; two were amnesic secondary to herpes simplex encephalitis; and one was amnesic secondary to closed-head injury. Extent of MTL lesion also varied; many of those patients who were willing and able to undergo structural MRI scans have been described in detail elsewhere (Allen et al., 2006; Feinstein et al., 2009; Gupta et al., 2009). The remaining patients are presumed to have damage limited to the MTL based on the available CT scans and etiologies. Neuropsychological test scores summarizing the severity of the patients' memory impairments are listed in Table $\mathbf{1 .}$ One comparison participant was matched to each patient on sex, age, handedness, and education. All participants granted informed consent prior to their experimental session.

\section{APPARATUS}

Experimental displays were presented on a 21-in Sony Trinitron Multiscan E540 monitor, and participants were seated in a chair approximately 36 in from the screen. Each participant's eye movements 
Table 1 | Details of participating neurological patients.

\begin{tabular}{|c|c|c|c|c|c|c|c|c|c|c|c|}
\hline \multirow[b]{2}{*}{ Patient } & \multirow[b]{2}{*}{ Etiology } & \multirow[b]{2}{*}{ Sex } & \multirow[b]{2}{*}{ Hd. } & \multirow[b]{2}{*}{ Onset } & \multirow[b]{2}{*}{ Age ('08) } & \multirow[b]{2}{*}{ Edu. (years) } & \multirow{2}{*}{$\begin{array}{l}\text { WAIS } \\
\text { FSIQ }\end{array}$} & \multicolumn{2}{|c|}{ WMS } & \multicolumn{2}{|c|}{ Volumes } \\
\hline & & & & & & & & GMI & DRI & $\mathrm{Hc}$ & TL \\
\hline 1606 & Anoxia & $M$ & $\mathrm{R}$ & 1990 & 61 & 12 & 91 & 66 & 61 & -2.11 & -3.99 \\
\hline 1846 & Anoxia & $\mathrm{F}$ & $\mathrm{R}$ & 1993 & 45 & 14 & 84 & 57 & 62 & -0.67 & -4.23 \\
\hline 1951 & HSE & M & $\mathrm{R}$ & 1980 & 56 & 16 & 121 & 75 & 53 & - & - \\
\hline 2308 & HSE & $M$ & $\mathrm{~L}$ & 1999 & 52 & 16 & 87 & 45 & 48 & - & - \\
\hline 2363 & Anoxia & M & $\mathrm{R}$ & 1998 & 52 & 16 & 98 & 73 & 74 & -1.70 & -2.64 \\
\hline 2563 & Anoxia & $M$ & $\mathrm{~L}$ & 2000 & 53 & 16 & 102 & 75 & 80 & - & - \\
\hline
\end{tabular}

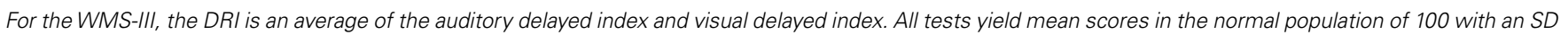

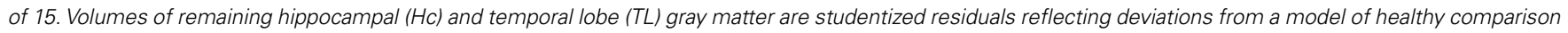
regional volumes (Allen et al., 2006).

CHI, closed-head injury; HSE, herpes simplex encephalitis; Hd., handedness; Educ., education; WAIS, Wechsler adult intelligence scale-III; FIQ, full-scale IQ; WMS, Wechsler memory scale-III; GMI, general memory index; DRI, delayed recall index.

were tracked using an Applied Science Laboratories model 504 remote tracker. This device tracked eye movements by reflecting infrared light off of the lens and the cornea of the eye, sampling eye position at $60 \mathrm{~Hz}$. The location and duration of fixations were determined in off-line analyses, described further in Section "Results."

\section{MATERIAL AND DESIGN}

Experimental displays were presented at a resolution of $1600 \times 1200$ pixels. Each display contained seven stimuli, including a centrally-presented sample item and six surrounding items arrayed in a hexagonal pattern centered on the sample. All surrounding items were at roughly the same eccentricity from the sample, i.e., approximately $8^{\circ}$ of visual angle.

The stimuli consisted of various simple shapes, and each visual dimension to be investigated was sampled at eleven successively spaced points along that dimension's continuum. Six different visual spectrums/dimensions were investigated, including: shape; luminance; proportion; spatial frequency; orientation; and linetilt. Each shape was encoded in a 391-pixel-square bitmap image with a medium gray background. These images subtended a maximum of $6.5^{\circ} \times 6.5^{\circ}$ of visual angle. Samples of the six types of search array are included in Figure 1.

\section{TASK}

Participants performed a simple visual search task in all conditions of the experiment, deciding whether a trial-specific target was present in an array of lures. Participants were informed that not all trials would include a matching item. Target presence or absence was indicated either by pressing "Yes" or "No" buttons on a keypad or by announcing the intended response to the experimenter who executed the button press on their behalf. Participants did not receive feedback regarding the accuracy of their responses. Search was performed either in the presence of a sample target or $6 \mathrm{~s}$ after the sample target for that trial had been removed. These two levels of the delay factor will be referred to as the "simultaneous" and "delayed" conditions. The stimuli were identical in these two conditions, and all stimulus types were administered in the simultaneous condition before the same order was repeated for the delayed condition. A substantial rest break was imposed between administrations of the two delay conditions. Stimulus types were presented to participants in a fixed order: linetilt; luminance; orientation; proportion; shape; and spatial frequency. Blocks contained 33 trials each.

In the simultaneous condition the participant fixated a cross in the center of the experimental display. When s/he had done so for an amount of time determined ad hoc and typically between 1 and $2 \mathrm{~s}$, the experimenter manually advanced the trial. The participant then viewed the sample stimulus item presented centrally for $2 \mathrm{~s}$ while six surrounding positions were masked with equiluminant RGB noise. Immediately thereafter, the display changed to unmask six items surrounding the still-present sample item; each lure was similar to the sample item in all but one respect, so that the set comprised a range along one visual dimension, e.g., orientation. An important design note is that the values of these six items were constant for a given dimension, i.e., the same six items always comprised the search array and the same six spatial locations were always filled (see Figure 4). Only the spatial positions of the search array items varied from trial to trial. The search array expired after $6 \mathrm{~s}$ and the participant was prompted to respond regarding the presence or absence of the target in the search array. Following that response, the end of the trial was signaled by the reappearance of the fixation screen. The six items comprising the search array were equally likely to be targets in target-present trials. A sample trial sequence for the simultaneous condition is presented in Figure 2.

The delayed condition was similar to the simultaneous condition in most respects, with the crucial difference being the introduction of a 6 s delay between exposure of the sample item and the presentation of the search array. After the 2 s study phase expired, the sample item was replaced by an equiluminant mask that persisted for about $4.5 \mathrm{~s}$. For the remainder of the $6 \mathrm{~s}$ delay phase, a cross was presented centrally and the participant was encouraged to fixate it. The small amount of random jitter in fixation onset was intended to interfere with attempts by the participant to anticipate the onset of the search array. Then, 6 s after the sample was removed, the six 


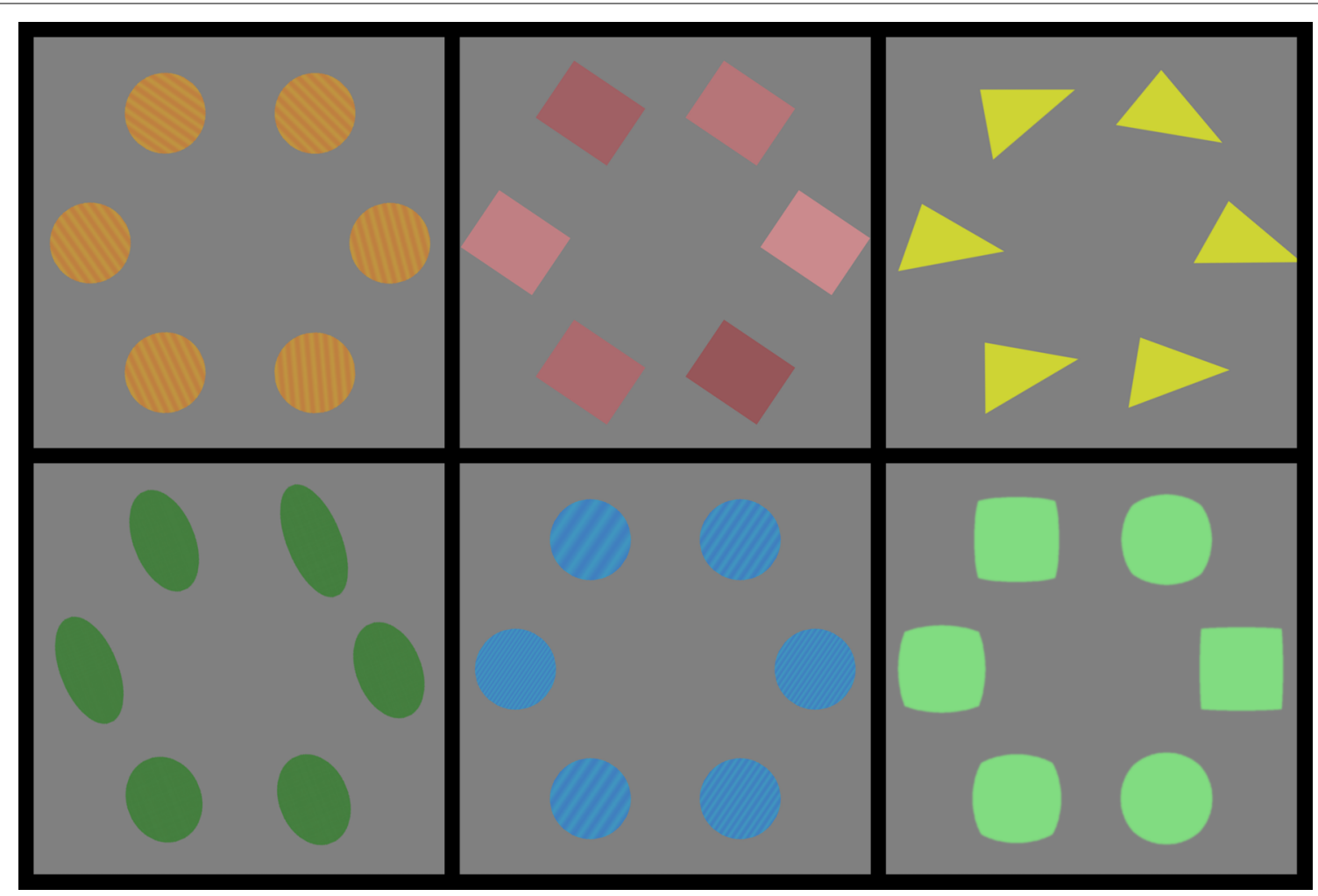

FIGURE 1 | Sample stimuli. Clockwise from top left: linetilt; luminance; orientation; shape; spatial frequency; proportion.

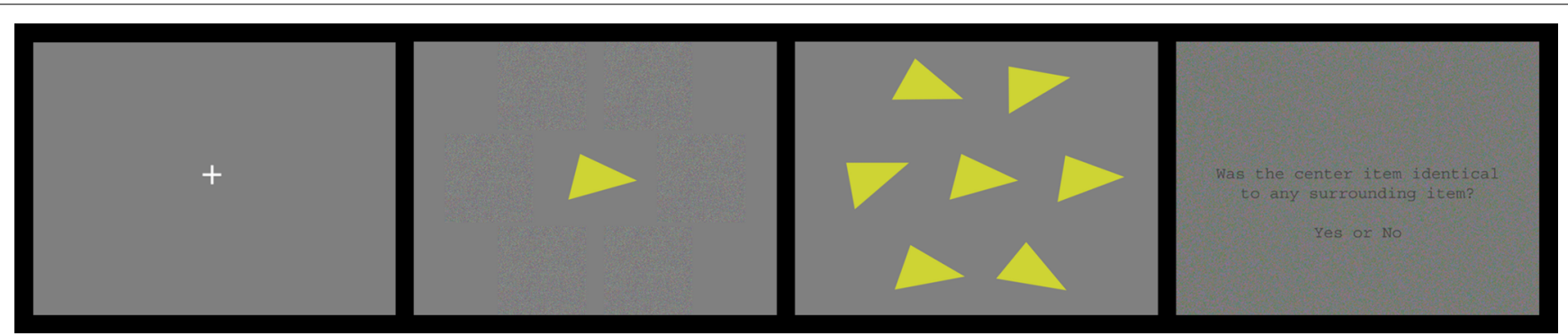

FIGURE 2 |Trial sequence for simultaneous presentation condition. From left to right: fixation; sample presentation (2s); sample persists and search array appears (6s); response screen.

peripheral items comprising the search array were presented. After a 6 s exposure to the search array, that display was replaced with a prompt for the participant to respond; responses were collected in the same fashion as the simultaneous condition. The six items comprising the search array were equally likely to be targets in target-present trials. A sample trial sequence for the delay condition is presented in Figure 3.

\section{RESULTS}

\section{BEHAVIORAL RESULTS}

Behavioral responses were collected from each participant, but some were lost due to computer malfunction. Of the 188 sessions conducted, 153 fully intact behavioral response records were available.
Behavioral sensitivity during search was uniformly poor, and all participants were biased to endorse displays as containing targets in all conditions. Tests of behavioral performance within each of the 24 unique combinations of factor levels revealed only three that were reliably above chance, and in 151 of 153 sessions the participant reported finding a target in the search array more frequently than chance would dictate. The proportion of correct responses was calculated for each participant in each condition and that measure of performance was examined using a repeated-measures ANOVA. Factors included MTL damage, delay, and stimulus type. Delay $[F(1,114)=9.40, p<0.01]$ and stimulus type $[F(5,114)=4.35, p<0.01]$ were both reliable predictors; MTL damage $[F(1,14)=3.74, p=0.07]$ and the interaction of delay 


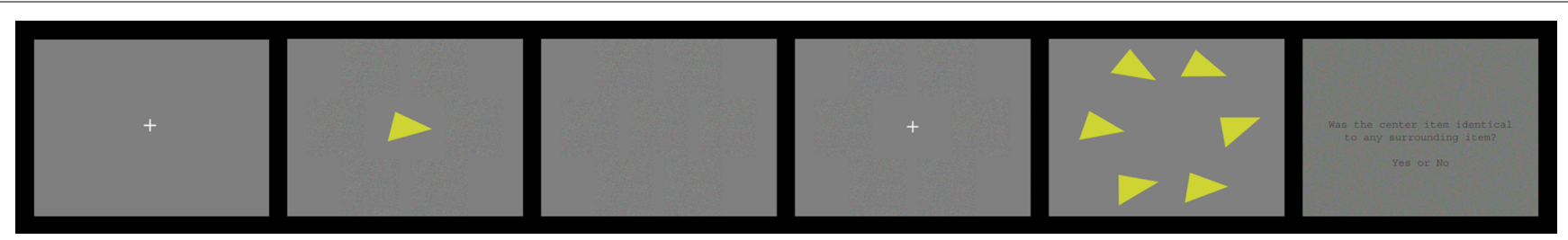

FIGURE 3 |Trial sequence for delayed condition. From left to right: fixation; sample presentation (2s); sample masked ( 4.5s); fixation ( 1.5s); search array presentation (6s); response screen.

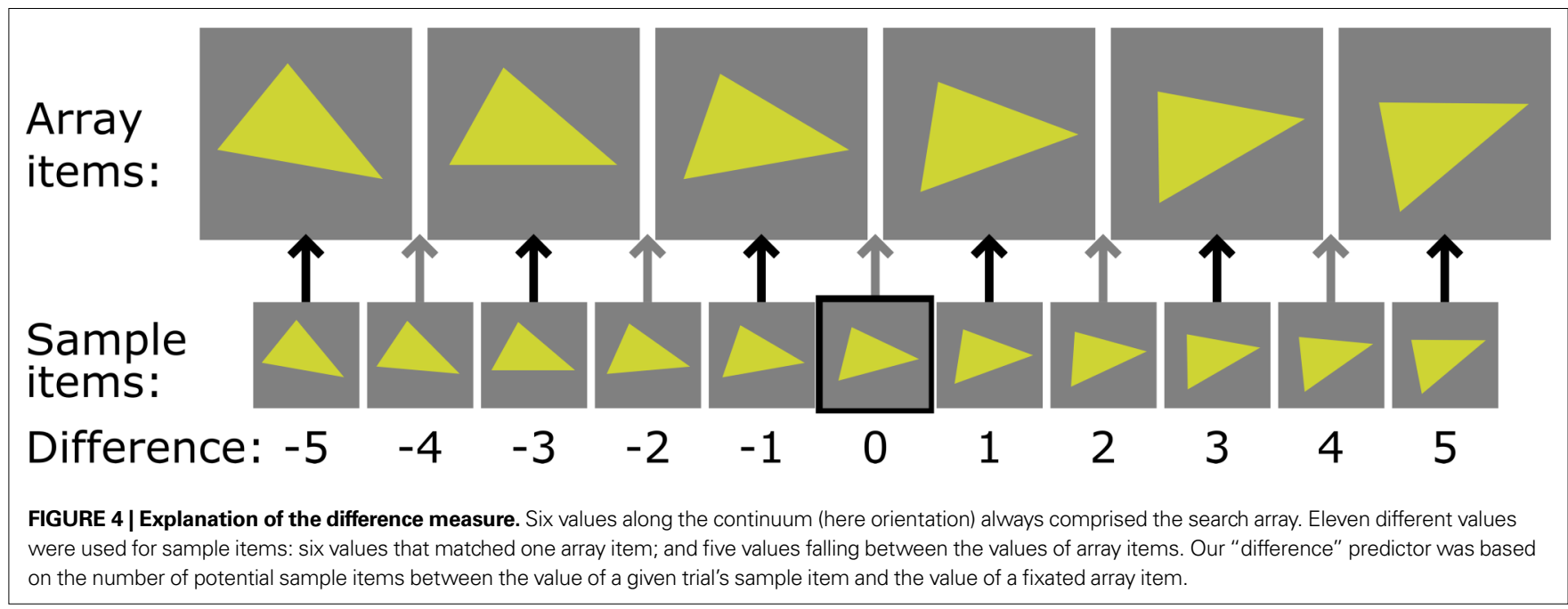

and stimulus type $[F(5,144)=2.068, p=0.07]$ were marginally significant. No other interactions approached reliability, including the interaction between delay and MTL damage.

Imposing a $6 \mathrm{~s}$ delay between sample presentation and the search array depressed performance overall, reducing the proportion of correct responses from 0.55 to 0.51. A marginal effect of MTL damage arose from a modest reduction in the overall performance of patients, whose proportion of correct responses was 0.52 relative to the 0.55 of comparisons. The marginal interaction of these two terms may have been spurious, as the performance of patients appears to have been bounded by the 0.50 chance level that both groups approached in the delayed trials, while comparison performance had farther to fall. Post hoc tests of performance with each stimulus type revealed that the orientation discrimination condition was easiest ( 0.57 prop. correct), and reliably better than linetilt, proportion, and shape discrimination (Tukey contrasts, each $p<0.05)$. Proportion correct for each combination of conditions is listed in Table 2.

\section{EYE-TRACKING RESULTS}

Eye-movement data were collected from each participant, but two participants were unable to complete the entire protocol owing to discontinued participation or chronic unavailability. Of the 192 blocks expected, 188 were completed. The qualitative outcomes of the analyses that follow were not altered by excluding the data of participants who did not complete the protocol, and therefore the entire data set was analyzed and reported.
Table 2 | Mean behavioral performance (proportion correct) and standard error in parentheses by stimulus condition, participant group, and delay condition.

\begin{tabular}{lcclll}
\hline $\begin{array}{l}\text { Stimulus } \\
\text { condition }\end{array}$ & \multicolumn{2}{c}{ MTL patient } & & \multicolumn{2}{c}{ Comparison } \\
\cline { 2 - 3 } \cline { 5 - 6 } & Simultaneous & Delayed & & Simultaneous & Delayed \\
\hline Linetilt & $0.49(0.02)$ & $0.51(0.07)$ & & $0.53(0.06)$ & $0.52(0.04)$ \\
Luminance & $0.57(0.08)$ & $0.48(0.02)$ & & $0.61(0.16)$ & $0.49(0.03)$ \\
Orientation & $0.56(0.07)$ & $0.53(0.03)$ & & $0.60(0.10)$ & $0.59(0.09)$ \\
Proportion & $0.53(0.06)$ & $0.51(0.02)$ & & $0.50(0.07)$ & $0.50(0.01)$ \\
Spat. Freq. & $0.56(0.09)$ & $0.48(0.03)$ & & $0.60(0.09)$ & $0.59(0.12)$ \\
Shape & $0.48(0.02)$ & $0.52(0.02)$ & & $0.57(0.07)$ & $0.48(0.03)$
\end{tabular}

No filtering of the eye-tracking data by behavioral performance was performed. Eye movements were characterized by the location of fixation within the display, which was divided for analysis into seven regions of interest (ROIs). ROIs were squares centered on the positions of the central sample item and the six peripheral items; the ROIs were all the same size and large enough to contain each type and instance of all presented stimuli. An eighth catch-all region covered the remainder of the display.

All collected eye-tracking data were required to surpass an objective, trial-level criterion for quality to be included in these analyses. The trial phase of interest was the 6s search-array presentation, and at least $3 \mathrm{~s}$ of total fixation time across all ROIs must have been 
recorded during that phase for the data collected in a specific trial to be included in any of the analyses presented here. Trials with less than $3 \mathrm{~s}$ of observed fixation time were relatively rare and presumed to reflect poor eye resolution. Approximately $2.2 \%$ of all fixations were discarded by this method, and another $1.1 \%$ of fixations were rejected for being shorter than $83 \mathrm{~ms}$, our duration criterion.

Fixation durations were a potentially rich source of information about the representations that participants maintained, but the data presented several challenges. First, corresponding to our expectations based on the eye-movement literature, the overall distribution of fixation durations exhibited pronounced positive skew. More powerful analytic techniques using all available data points were available if the data were distributed approximately normally, and for that reason we log-transformed the fixation-duration data. This action had the desired effect of producing an approximately normal distribution. Second, per-participant distributions of fixation durations were observed to vary reliably $[F(15,65577)=181.67, p$ is vanishingly small], and this baseline variation had the potential to interfere with any analysis involving effects of the experiment's independent variables. Linear mixed models (LMMs) offer a means of controlling for differences between unique sources of data. In our LMM-based analysis, fixation data were nested within participants, and the baseline variation between participants was treated as a normally-distributed random effect. Together, the log transform and LMM controlled for important differences in the fixation data. All models were fit using $R$ 2.9.1 and its lme4 library. Third, fixations arose organically from search behavior, and therefore the number of fixations made to each type and value of item was not fixed. Further, the frequency of observations at each degree of perceptual difference was constrained to be uneven by our design. Combined, these two influences resulted in many more fixations to items most similar to the sample, and less to items most dissimilar to the sample $[F(1,174)=530.84, p$ is vanishingly small $]$. No attempt was made to correct for this imbalance, but the effects described later were driven by differences at the most frequently sampled levels of perceptual difference, i.e., 0,1 , and 2 .

Our initial LMM approach used the degree of difference between a lure and the sample as a continuous predictor variable for the fixation duration outcome variable; delay and MTL damage were treated as discrete predictors. Individual participants were treated as a random effect, with each participant contributing a unique offset to the model's intercept term.

The simple LMM model indicated several interesting trends in the data, but visual inspection of the overall fitted curves suggested that the model might be improved by including quadratic and cubic transforms of the predictor indicating a lure's degree of difference from the sample target. Additionally, a "hinge" or "elbow" between difference levels 5 and 6 in the fixation duration data inspired inclusion of a cubic spline term in our model with a single knot at difference level 5. Inclusion of the cubic spline reliably improved model fit and visual fit of the derived curves to the mean data [95\% highest posterior density interval (HPDI) did not include 0] and improved model fit substantially $\left[\chi^{2}(10)=3131.5\right.$, $p=0$; non-spline model AIC $=110363, \mathrm{BIC}=110391$ vs. splineincorporating model $\mathrm{AIC}=107358, \mathrm{BIC}=107477]$. Owing to the improved fit, statistics and plotted estimates that follow derive from the best-fit model.
Parametric enhancements notwithstanding, all fitted models indicated that the following predictors and interactions were reliable: perceptual difference; delay condition; perceptual difference-by-delay condition; and perceptual difference-by-delay condition-by-MTL status. Both groups of participants showed a robust relationship between the duration of their average fixations and the similarity of fixated lures to the sample item (see Figure 5). Further, comparisons and patients exhibited a statistically indistinguishable manifestation of this effect in the simultaneous presentation condition (quadratic and cubic transforms of similarity predictor reliable by $95 \% \mathrm{HPDI}$ ). Items more similar to a trial's sample target were fixated longer than items less similar to the sample (see Figure 6A).

Fixation durations in the delayed condition were reliably different from those in the simultaneous condition. Comparison participants made reliably longer fixations $(-38 \mathrm{~ms})$ when the delay was imposed (delay predictor reliable by $95 \%$ HPDI), while patients did not (delay-by-MTL-lesion interaction not reliable by 95\% HPDI). Additionally, the modulation of fixation duration by similarity changed in the delay condition. The effect was exaggerated in comparisons, with even longer fixations to more similar items (delay-by-difference interaction reliable by 95\% HPDI, positive value), but attenuated in patients (delay-by-difference-by-MTL status three-way interaction reliable by $95 \%$ HPDI, negative value) (see Figure 6B). Patients still showed a graded effect in that the slope of the relationship was reliably different from 0 , but that slope was shallower than the slope observed in the no-delay condition (similarity-by-delay-by-MTL-lesion interaction predictor reliable by $95 \%$ HPDI with positive parameter value), contrasting with the effect seen in comparisons.

This marked difference in patient fixation duration was subjected to further scrutiny by fitting unique models to the fixation duration data of each participant. These models were similar to the full model described earlier, but lacked the participant-level predictors, e.g., memory status. Thresholds for reliability were adjusted across a wide range in order to investigate which participants

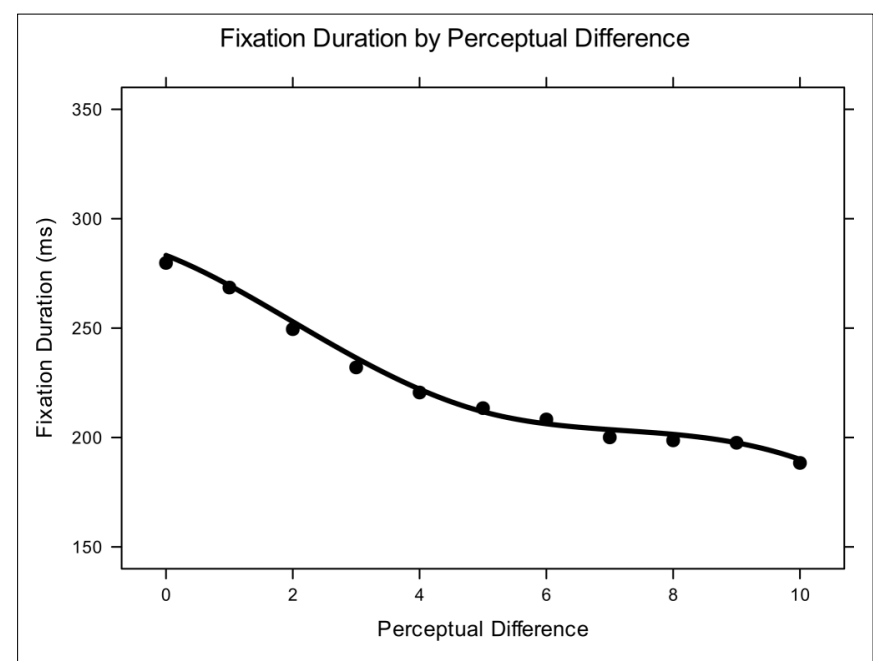

FIGURE 5 | Overall effect of perceptual difference on fixation duration.

Overall means and model-fitted curve. 

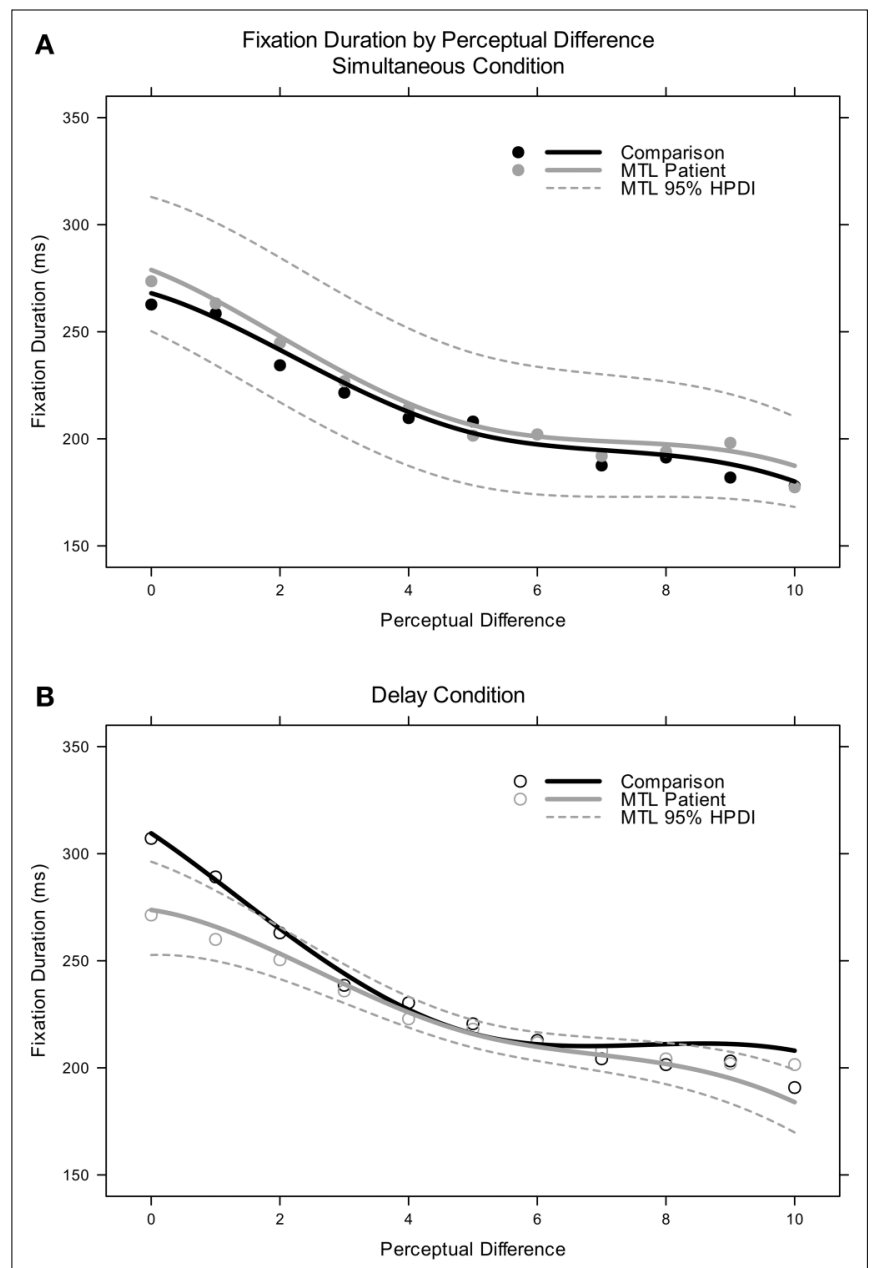

FIGURE 6 | Fixation duration, overall means and model-fitted curves: (A, top) simultaneous condition; (B, bottom) delayed condition. In (A), 95\% HPDI curves reflect the main effects of perceptual difference and MTL damage, while in (B), the HPDI curves reflect the interactions of those terms with delay.

exhibited the patterns observed in the aggregate data above: very strict thresholds, i.e., 0.99 , revealed three patients $(0002,1951,2144)$ and no comparisons who showed attenuation of the similarityfixation-duration effect in the delayed condition; very lax thresholds, i.e., 0.5, suggested that seven of eight patients (all but 2363) and two comparisons exhibited the same pattern. Thus, while the statistical index of attenuation may have been driven principally by a subset of the patients, seven of eight patients showed this pattern to some extent. Only two of eight comparison participants showed the same pattern even using the same extremely lax threshold for reliability. Effects of temporal lobe and hippocampal atrophy were evaluated qualitatively among the four patients for whom such data were available, but did not reveal any obvious trends.

\section{DISCUSSION}

MTL damage has long been thought to leave most cognitive faculties other than long-term memory undiminished, and thus performance requiring only short-delay discrimination of simple visual stimuli might reasonably be expected to be unimpaired. In contrast to that expectation and more in line with recent findings of deficits on short-delay tasks after MTL damage, in the current visual search task the introduction of an unfilled 6s delay between sample and search wrought important changes on the eye movement behavior of MTL-lesion patients during visual search performance. These eye movement effects reveal the rapid change in the representation of items over the delay interval in patients with MTL damage.

Impairments in delayed discrimination tasks employing simple visual stimuli have been reported previously (Prisko, 1963; Sidman et al., 1968; Olson et al., 2006a). But of these previous reports, the dataset with the richest information about the nature of the impairment (Sidman et al., 1968) was poorly characterized statistically and lacking in adequately-matched comparison participants. The current work extends those findings by addressing such shortcomings and by providing a window into the delay-induced changes in representations using eye-movement behavior. Eye-movement data in general and fixation-duration data in particular provided powerful outcome measures because of their sheer richness; many items were sampled on each trial, permitting statistical inference about the relationship between fixation duration and lure-sample similarity based on data from each of the sampled items during every trial. By contrast, typical behavioral responses indicating the choice of the target item in each test array yield only a single binary datum per trial. Notably, tuning functions of greater sensitivity than those reported by Sidman et al. (1968) have been reported after short delays, but in the field of visual short-term memory rather than long-term memory. The work of Zhang and Luck $(2008,2009)$ deserves particular attention, as does their hypothetical cognitive mechanism of visual short-term memory, i.e., a fixed number of registers with good but limited precision. Whether the number or precision of these hypothesized registers can be modulated by MTL lesion remains an open question.

Eye movements revealed much about the underlying representations driving task performance. Longer fixations were made to stimuli most similar to the target, and shorter fixations were made to items resembling the target to a lesser degree, reminiscent of the behavioral results reported for delayed discrimination among similar shapes by Sidman et al. (1968). All of our participants exhibited this trend irrespective of the delay condition, but a marked difference emerged between comparisons and patients after a $6 \mathrm{~s}$ delay. Although the effect of similarity on fixation duration was statistically equivalent for patients and comparisons in the simultaneous condition, the 6 s delay resulted in a reduction of this effect in patients whereas the same condition enhanced the effect for comparison participants.

One important caveat that must accompany our findings is that brain lesions of any kind typically impede processing speed, attention, and task engagement. We did not collect data from other brain-damaged patients for purposes of comparison, so the possibility that our findings are not specific to MTL damage must be acknowledged. However, the absence of group differences for either the behavioral or eye-movement results in the simultaneous condition suggest that the patients were not globally disadvantaged at performing this task relative to healthy comparisons, nor were their eye movements abnormal in any general way. 
Deficits in short-delay tasks in MTL-lesion patients have now been reported multiple times with complex stimulus materials or in conditions with high relational memory loads. But the deficit seen here was evoked with very simple materials, where deficits have not often been observed. No performance deficits were observed in one line of experiments that employed forced-choice test formats and lures very similar to the target, but these experiments did not vary the similarity of the lures parametrically (Holdstock et al., 2002, 2005). Intact performance was also reported for discrimination among several simultaneously-available stimuli when based on simple dimensions such as color, size, or shape, but the task in those reports was an odd-man-out design, in which all lures were (necessarily) the same (Lee et al., 2005; Barense et al., 2007). There is another report of performance deficits after brief delays for color representations, even though lure-target similarity was not varied (Olson et al., 2006a). One promising investigation that parametrically varied the similarity of lure faces to the target, using morphing, did find behavioral choices to be sensitive to target-lure similarity, but unlike the current findings no interaction was found between MTL damage and this similarity effect (Ezzyat and Olson, 2008). We believe that the unusual sensitivity of the current paradigm to possible deficits was due to the use of highly similar lures, systematic variation in similarity of lures to the target, and rich eye movement measures of the relationship of viewing to lure-target similarity as an index of target representation quality.

A critical contribution of the current data is evidence of representation decay over time in patients with MTL damage. We suggest that visual stimuli that do not readily afford a verbal code sufficient to permit discrimination from among similar items are held in a form of representation that apparently decays or degrades when MTL is damaged during even the short delays used here. Whatever decayed representation remains appears to anchor subsequent behavior. That is, the fact that both previously-reported behavioral (Sidman et al., 1968) and our current eye movement responses were not distributed randomly but rather retained a systematic relation to target-lure similarity indicates that there is not a complete loss of representation; instead it indicates that degraded representations guide performance. The current findings argue for a critical role for the MTL in insulating simple non-verbal representations from such representation decay.

\section{REFERENCES}

Allen, J. S., Tranel, D., Bruss, J., and Damasio, H. (2006). Correlations between regional brain volumes and memory performance in anoxia. $J$. Clin. Exp. Neuropsychol. 28, 457-476.

Barense, M. D., Gaffan, D., and Graham, K. S. (2007). The human medial temporal lobe processes online representations of complex objects. Neuropsychologia 45, 2963-2974.

Buffalo, E. A., Reber, P. J., and Squire, L. R. (1998). The human perirhinal cortex and recognition memory. Hippocampus 8, 330-339.

Cave, C. B., and Squire, L. R. (1992). Intact verbal and nonverbal short-term memory following damage to the

Whatever its form and timecourse, decay of representation is an observed phenomenon, not an ultimate explanation (McGeoch, 1932). What accounts for the decay in representation seen after MTL damage, or the role of MTL in protecting against it? One possibility is that healthy comparison participants are able to recruit MTL structures in service of coding items that would be difficult to differentiate otherwise (c.f., Norman and O'Reilly, 2003); i.e., for forming more distinctive representations (or pattern separation), critical here due to the use of visually similar materials that do not have verbal labels useful for discrimination. There are a number of mechanisms that might underlie such an ability. One such mechanism might entail the ability of healthy comparison participants to create, revise, and refine distinctive encodings based on the capacity for forming new enduring memories of the various stimuli within and across trials; amnesia due to MTL lesions would prevent the use of this longer-duration memory to help in creating rich encodings. A second, related mechanism might involve the ability of healthy comparison participants to deploy memory in a flexible manner, e.g., to creating encodings for each target stimulus by taking into account an item's relative value with respect to the stimulus continuum for each dimension; amnesia due to MTL lesions would prevent the use of such a flexible, relational form of memory representation (Cohen and Eichenbaum, 1993; Eichenbaum et al., 1994; Eichenbaum and Cohen, 2001).

Further studies should examine these and other possible mechanisms for the role of MTL in this task. Nevertheless, the findings reported here document the degradation or decay of representations of simple stimuli over even the brief delays employed here when the MTL is damaged. Accordingly, regardless of the precise mechanism of its action, the MTL must participate in the representation of simple stimuli not only over extended delays, but also over very short intervals not traditionally considered within its purview.

\section{ACKNOWLEDGMENTS}

This work was supported by NIMH grant MH062500 to Neal J. Cohen, program project grant NINDS NS19632 to Daniel Tranel, and NIDA grant DA022549 to Daniel Tranel. The authors would like to acknowledge the assistance of undergraduate research assistant David Sacks and research programmer Jake Gibbons at the University of Illinois.

of the hippocampal memory system. Behav. Brain Sci. 17, 449-472. 2, 151-163.

Cohen, N. J., and Eichenbaum, H. (1993). Memory, Amnesia and the Hippocampal System. Cambridge, MA: MIT Press.

Cohen, N. J., and Squire, L. R. (1980). Preserved learning and retention of pattern-analyzing skill in amnesia - dissociation of knowing how and knowing that. Science 210, 207-210.

Eichenbaum, H., and Cohen, N. J. (2001). From Conditioning to Conscious Recollection: Memory Systems of the Brain. Oxford: Oxford University Press.

Eichenbaum, H., Otto, T., and Cohen, N. J. (1994). 2 Functional components
Ezzyat, Y., and Olson, I. R. (2008). The medial temporal lobe and visual working memory: comparisons across tasks, delays, and visual similarity. $\operatorname{Cog} n$. Affect. Behav. Neurosci. 8, 32-40.

Feinstein, J. S., Rudrauf, D., Khalsa, S. S., Cassell, M. D., Bruss, J., Grabowski, T. J., and Tranel, D. (2009). Bilateral limbic system destruction in man. J. Clin. Exp. Neuropsychol. 17, 1-19. Cohen, N. J., Bechara,A., and Tranel, D. (2009). Declarative memory is critical for sustained advantageous complex decision-making. Neuropsychologia 47, 1686-1693.
Gupta, R., Duff, M. C., Denburg, N. L.,
Hannula, D. E., Ryan, J. D., Tranel, D., and Cohen, N. J. (2007). Rapid onset relational memory effects are evident in eye movement behavior, but not in hippocampal amnesia. J. Cogn. Neurosci. 19, 1690-1705.

Hannula, D. E., Tranel, D., and Cohen, N. J. (2006). The long and the short of it: relational memory impairments in amnesia, even at short lags. J. Neurosci. 26, 8352-8359.

Hartley, T., Bird, C. M., Chan, D., Cipolotti, L., Husain, M., VarghaKhadem, F., and Burgess, N. (2007). The hippocampus is required for short-term topographical memory in humans. Hippocampus 17, 34-48. 
Holdstock, J. S., Gutnikov, S. A., Gaffan, D., and Mayes, A. R. (2000). Perceptual and mnemonic matching-to-sample in humans: contributions of the hippocampus, perirhinal and other medial temporal lobe cortices. Cortex 36, 301-322.

Holdstock, J. S., Mayes, A. R., Gong, Q. Y., Roberts, N., and Kapur, N. (2005). Item recognition is less impaired than recall and associative recognition in a patient with selective hippocampal damage. Hippocampus 15 , 203-215.

Holdstock, J. S., Mayes, A. R., Roberts, N., Cezayirli, E., Isaac, C. L., O’Reilly, R. C., and Norman, K.A. (2002). Under what conditions is recognition spared relative to recall after selective hippocampal damage in humans? Hippocampus 12, 341-351.

Lee, A. C. H., Buckley, M. J., Pegman, S. J., Spiers, H., Scahill, V. L., Gaffan, D., Bussey, T. J., Davies, R. R., Kapur, N., Hodges, J.R., and Graham, K. S. (2005). Specialization in the medial temporal lobe for processing of objects and scenes. Hippocampus 15, 782-797.

McGeoch,J.(1932). Forgetting and the law of disuse. Psychol. Rev. 39, 352-370.
Nichols, E. A., Kao, Y. C., Verfaellie, M., and Gabrieli, J. D. E. (2006). Working memory and long-term memory for faces: evidence from fMRI and global amnesia for involvement of the medial temporal lobes. Hippocampus 16, 604-616.

Norman, K. A., and O’Reilly, R. C. (2003). Modelinghippocampal and neocortical contributions to recognition memory: a complementary-learning-systems approach. Psychol. Rev. 110, 611-646.

Olson, I. R., Moore, K. S., Stark, M., and Chatterjee,A. (2006a).Visual working memory is impaired when the medial temporal lobe is damaged. J. Cogn. Neurosci. 18, 1087-1097.

Olson, I. R., Page K, Moore, K. S., Chatterjee, A., and Verfaellie, M. (2006b). Working memory for conjunctions relies on the medial temporal lobe. J. Neurosci. 26, 4596-4601.

Prisko, L. (1963). Short-Term Memory in Focal Cerebral Damage. Unpublished doctoral dissertation, McGill University, Montreal.

Ryan, J. D., Althoff, R. R., Whitlow, S., and Cohen, N. J. (2000). Amnesia is a deficit in relational memory. Psychol. Sci. 11, 454-461.
Ryan, J. D., and Cohen, N. J. (2004a) The nature of change detection and online representations of scenes. J. Exp. Psychol. Hum. Percept. Perform. 30, 988-1015.

Ryan, J. D., and Cohen, N. J. (2004b) Processing and short-term retention of relational information in amnesia. Neuropsychologia 42, 497-511.

Scoville, W. B., and Milner, B. (1957). Loss of recent memory after bilateral hippocampal lesions. J. Neurol. Neurosurg. Psychiatry 20, 11-21.

Sidman, M., Stoddard, L. T., and Mohr, J.P. (1968). Some additional quantitative observations of immediate memory in a patient with bilateral hippocampal lesions. Neuropsychologia 6, 245-254.

Warrington, E. K., and Baddeley, A. D. (1974). Amnesia and memory for visual location. Neuropsychologia 12, 257-263.

Wickelgren, W. A. (1968). Sparing of short-term memory in an amnesic patient: implications for strength theory of memory. Neuropsychologia 6, 235-244.

Zhang, W., and Luck, S. J. (2008). Discrete fixed-resolution representations in visual working memory. Nature 453, 233-235.

Zhang, W., and Luck, S. J. (2009). Sudden death and gradual decay in visual working memory. Psychol. Sci. 20, 423-428.

Conflict of Interest Statement: The authors declare that the research was conducted in the absence of any commercial or financial relationships that could be construed as a potential conflict of interest.

Received: 17 December 2009; paper pending published: 18 January 2010; accepted: 10 April 2010; published online: 18 May 2010.

Citation: Warren DE, Duff MC, Tranel D and Cohen NJ (2010) Medial temporal lobe damage impairs representation of simple stimuli. Front. Hum. Neurosci. 4:35. doi: 10.3389/fnhum.2010.00035

Copyright $\odot 2010$ Warren, Duff, Tranel and Cohen. This is an open-access article subject to an exclusive license agreement between the authors and the Frontiers Research Foundation, which permits unrestricted use, distribution, and reproduction in any medium, provided the original authors and source are credited. 